\title{
HPRT1 activity loss is associated with resistance to thiopurine in ALL
}

\author{
Fan Yang ${ }^{1,2,3, *}$, Houshun Fang ${ }^{2,3, *}$, Dan Wang ${ }^{2,3, *}$, Yao Chen ${ }^{2,3}$, Yonggong Zhai ${ }^{1}$, Bin- \\ Bing S. Zhou ${ }^{2,3,4}$ and Hui $\mathbf{L i}^{2,3,4}$ \\ ${ }^{1}$ Beijing Key Laboratory of Gene Resource and Molecular Development, College of Life Sciences, Beijing Normal University, \\ Beijing, China \\ ${ }^{2}$ Key Laboratory of Pediatric Hematology and Oncology Ministry of Health, Department of Hematology and Oncology, \\ Shanghai Children's Medical Center, Shanghai Jiao Tong University School of Medicine, Shanghai, China \\ ${ }^{3}$ Pediatric Translational Medicine Institute, Shanghai Jiao Tong University School of Medicine, Shanghai, China \\ ${ }^{4}$ Department of Pharmacology and Chemical Biology, School of Basic Medicine and Collaborative Innovation Center for \\ Translational Medicine, Shanghai Jiao Tong University School of Medicine, Shanghai, China \\ *These authors contributed equally to this work \\ Correspondence to: Hui Li, email: huili1219@163.com
}

Bin-Bing S. Zhou, email: binbing_s_zhou@yahoo.com

Keywords: thiopurine; leukemia; drug resistance; HPRTI mutation; purine metabolism

Received: August 02, $2017 \quad$ Accepted: December 03, $2017 \quad$ Published: December 19, 2017

Copyright: Yang et al. This is an open-access article distributed under the terms of the Creative Commons Attribution License 3.0 (CC BY 3.0), which permits unrestricted use, distribution, and reproduction in any medium, provided the original author and source are credited.

\section{ABSTRACT}

Acute lymphoblastic leukemia (ALL) is an aggressive hematological tumor resulting from the malignant transformation of lymphoid progenitors. Thiopurine is a widely used drug in the maintaining treatment of ALL. After a period of chemotherapy, $20 \%$ of pediatric patients and over $50 \%$ of adult patients will relapse. To investigate the mechanisms of drug resistance in vitro, we established the thiopurine resistant cell lines Reh-6MPR (6-MP Resistant cell) and Reh-6TGR (6-TG Resistant cell) by stepwise selection of the ALL cell line Reh. Cell viability assay revealed that 6MPR and 6TGR cells were almost $\mathbf{1 0 0 0}$-fold more resistant to thiopurine comparing with the control Reh cells, and thiopurine conversion was significantly impaired in the resistant cells. Mechanistically, a same novel hypoxanthine phosphoribosyl transferase 1 (HPRT1) mutation c.495_496insA (p.V165fs) was found by whole exome sequencing in both resistant cells. The HPRT1 mutation dramaticly decreased the production of $\left[{ }^{13} \mathrm{C}_{5}{ }^{15} \mathrm{~N}_{4}\right]-$ IMP from $\left[{ }^{13} \mathrm{C}_{5}{ }^{15} \mathrm{~N}_{4}\right]$-hypoxanthine ( $\mathrm{HX}$ ), showed a loss-of-funciton mechanism. Notably, re-expression the wildtype HPRT1 in Reh-6MPR cell can reverse the drug resistance and thiopurine conversion in Reh-6MPR cells. These results highlight the importance of HPRT1's activity in thiopurine resistance.

\section{INTRODUCTION}

Acute lymphoblastic leukemia (ALL) is the most common childhood cancer. The survival rates of childhood ALL have been improved with the development of riskstratified combination chemotherapy. Though patients with relapsed ALL generally receive more intense treatment, relapsed ALL is still the leading cause of childhood cancer death, with cure rates of less than $40 \%$, owing to intrinsic drug resistance [1-4]
The thiopurines 6-mercaptopurine (6-MP) and 6-thioguanine (6-TG) are key drugs in the treatment of ALL, administered to induce remission and used during maintenance therapy [5]. Both of them are prodrugs, which require intracellular conversion into active compounds by purine salvage pathway enzyme hypoxanthine phosphoribosyl transferase 1 (HPRT1). 6-MP and 6-TG are converted by HPRT1 into thioinosine monophosphate (TIMP) and thioguanosine monophosphate (TGMP), respectively, and subsequently into the active cytotoxic 
thioguanine nucleotides (TGNs), which are incorporated into DNA during DNA replication and exhaust the DNA repair machinery by inducing repeated mismatch repair. Therefore the activity of HPRT1 can affect the therapeutic efficacy of these drugs [6]. However, which kind of HPRT1 mutation will cause drug resistance is not fully understand.

To investigate the mechanisms of drug resistance in ALL, we established the resistant cell lines Reh-6MPR (6-MP Resistant cell) and Reh-6TGR (6-TG Resistant cell) by inducing Reh cells with 6-MP or 6-TG, and we found a novel HPRT1 mutation c.495_496insA (p.V165fs) in these two resistant cells by whole exome sequencing. In control cells, HPRT1 convert the thiopurines to TIMP and TGMP. But in the resistant cell lines, the function of HPRT1 is impaired and the metabolism of thiopurines is defected. The 6MPR and 6TGR cells shows 1000fold more resistant to thiopurine comparing with control Reh cells. Re-expression of wildtype HPRT1 in these cells could reverse the drug resistance phenotype. Our results indicate a new resistance mechanism and potential therapeutic strategies to overcome thiopurine resistance.

\section{RESULTS}

\section{Establishment of the thiopurine resistance cell lines Reh-6MPR and Reh-6TGR}

To investigate the mechanisms of cellular resistance to thiopurine in ALL, we induced thiopurine-resistant cell lines from the Reh human ALL cells. Reh cells were selected by stepwise increasing concentrations of 6-MP or 6-TG during a 3-months period. Finally, we established two resistant cell lines Reh-6MPR and Reh6TGR. Reh-6MPR and Reh-6TGR cells showed stable resistant phenotypes at least for 6 months after continued growth without drugs. Cell viability assay indicated that Reh-6MPR cells were 968- and 1161-fold more resistant to 6-MP and 6-TG respectively, compared with control Reh cells culturing for the same long time. Similarly, the Reh-6TGR cells were 699- and 956- fold more resistant to 6-MP and 6-TG than control Reh respectively (Table 1, Figure 1A and 1B). Interestingly, the Reh-6MPR and Reh-6TGR cells were still as sensitive as control Reh cells to other chemotherapeutic drugs that are used in ALL treatment, such as methotrexate (MTX) and cytosine arabinoside (AraC) (Table 1, Figure 1C and 1D). That leads to the suggestion that the resistance of these two cell lines is specific to 6-MP and 6-TG.

Thiopurines exert their cytotoxicity primary by incorporating TGNs into DNA, leading to DNA damage response (DDR) and apoptosis. We found that much less Reh-6MPR and Reh-6TGR cells went apoptosis when inducing by thiopurines than the Reh cells (Figure 1E and $1 \mathrm{~F})$. Moreover, the expression of the DDR biomarkers $\gamma$-H2AX, the phospho-CHK2 (T68) and the apoptosis biomarker cleaved PARP were much lower in the resistant cell line Reh-6MPR than those in control Reh cells, consistent with reduced DNA damage (Figure 1G).

\section{The thiopurine metabolism was impaired in the resistant cells}

To determine how the resistant cells avoid thiopurine-induced DDR, we analyzed the thiopurine metabolism in resistant cells and control Reh cells. 6-MP and 6-TG are converted by HPRT1 into TIMP and TGMP, respectively, subsequently converted by the other purine salvage pathway enzymes into the active TGNs, which incorporated into DNA (Figure 2A). We used LC-MS to measure the levels of 6-MP, 6-TG, TIMP and TGMP, which representing thiopurine metabolism in cells. The results revealed that the 6-MP or 6-TG were accumulated in the resistant cells (Figure 2B and 2C), and the thiopurine metabolites TIMP and TGMP were markedly reduced in resistant cells compared with Reh cells (Figure 2D and 2E). These results suggested that the resistant cells can not convert thiopurine into active TGNs and exert resistance to drug-induced DDR and apoptosis.

\section{HPRT1 loss of function mutation confers to the resistance to thiopurines}

To explore the mechnism of the drug resistance in Reh-6MPR and Reh-6TGR cells, we performed whole exome sequencing in the resistant and control Reh cells (Supplementary Tables 1 and 2). Interestingly, we found a novel HPRT1 mutation at the same site [c.495_496insA (p.V165fs)] in Reh-6MPR and Reh-6TGR cells. The mutation causes the HPRT1 frameshift and premature termination. To confirm the results, we performed sanger sequencing in the resistant and control Reh cells. As shown in Figure 3A, HPRT1 was mutated in Reh-6MPR and Reh-6TGR cells but wildtype in control Reh cells. To our knowledge, this mutation has not been reported before [7, 8]. Furthermore there were no thiopurine S-methyltrasferase gene (TPMT) [9], phosphoribosyl pyrophosphate synthetase 1 gene (PRPS1) [10] and cytosolic 5'-nucleotidase II gene (NT5C2) [11] mutations in Reh-6MPR and Reh-6TGR cells (Supplementary Tables 1 and 2), which previously shown to be associated with thiopurine resistance in ALL.

To test the functional effect of HPRT1 mutaion, we measured the HPRT1 activity in the Reh-6MPR, Reh-6TGR and Reh cells by following the generation of $\left[{ }^{13} \mathrm{C}_{5},{ }^{15} \mathrm{~N}_{4}\right]$-IMP from $\left[{ }^{13} \mathrm{C}_{5},{ }^{15} \mathrm{~N}_{4}\right]$-hypoxanthine (HX). The results showed that the HPRT1 activity was significantly decreased in the resistant cells, consistent with the HPRT1 mutation is a loss of function mutation (Figure 3B). According to the protein structure study, there were 11 active sites in the conserved Phosphoribosyl transferase (PRT)-type I domain [12]. The frameshift 
Table 1: IC50 of anticancer drugs in Reh cells and resistant cells

\begin{tabular}{lccccc}
\hline \multirow{2}{*}{ Drugs } & \multicolumn{3}{c}{ IC50 $(\boldsymbol{\mu g} / \mathbf{m L})$} & Relative resistance & ${ }^{\text {b }}$ Relative resistance \\
\cline { 2 - 4 } & Reh & Reh-6MPR & Reh-6TGR & & \\
\hline 6-MP & $0.18 \pm 0.02$ & $174.23 \pm 16.75^{* * *}$ & $125.73 \pm 3.54^{* * *}$ & 968 & 699 \\
6-TG & $0.09 \pm 0.01$ & $104.50 \pm 2.79^{* * *}$ & $86.05 \pm 5.82^{* * *}$ & 1161 & 956 \\
MTX & $0.005 \pm 0.0006$ & $0.0039 \pm 0.0001$ & $0.004 \pm 0.0002$ & 0.78 & 0.80 \\
AraC & $0.0024 \pm 0.0001$ & $0.0018 \pm 0.0002$ & $0.0016 \pm 0.0001$ & 0.75 & 0.67 \\
\hline
\end{tabular}

Values are given as mean \pm SD. IC50 of resistant cells to 6-MP and 6-TG differed significantly from Reh cells, ${ }^{* * *} P<0.001$ compared with Reh group. ${ }^{a}$ Relative resistance was obtained by dividing the IC50 mean value of Reh cells by the IC50 value of Reh-6MPR cells; ${ }^{b}$ Relative resistance was obtained by dividing the IC50 mean value of Reh cells by the IC50 value of Reh-6TGR cells.

from V165 could only influence the last active site. Our data indicated that the last active site is essential for the function of PRTases type I domain or there is still another domain, which is essential for the function of HRPT1. Furthermore, we measured the de novo purine biosynthesis pathway's activity. To our surprise, the nucleotides IMP, 5-amimoimidazole-4- carboxamide-1- $\beta$-d-ribofuranoside (AICAR), Inosine and HX were dramatically increased in the resistant cell lines, suggested that the de novo purine synthesis pathway was hyper activated in the resistant cells (Figure 3C-3F).

Meanwhile, we tested the expression of multidrug resistance gene 1 (MDR1) $[13,14]$ which is a direct active transporter for a variety of drugs. There was no significant difference between Reh cells and the resistant cells, consistent with 6-MP or 6-TG accumulation in resistant cells (Figure 3G). Reading frame shift and premature termination usually cause mRNA premature degradation, so we also measured the mRNA and protein levels of HPRT1 in the resistance cells. As shown in Supplementary Figure 1A and 1B, the mRNA and protein level of HPRT1 were both significantly reduced in the Reh-6MPR and Reh-6TGR cells compared to control Reh cells. These results suggested that the thiopurine uptake is normal but the thiopurine conversion is impaired by HPRT1 loss of function mutation in the resistant cells.

\section{Re-expression of wildtype HPRT1 can reverse the thiopurine resistant phenotype in Reh-6MPR cells}

To test whether loss the activity of HPRT1 is specifically contribute to the drug resistance phenotype, we transfected wildtype or mutant HPRT1 expression cassete to the resistance cell line Reh-6MPR (Figure 4A). Expression of wildtype HPRT1 could reverse the thiopurine resistant phenotype of resistant cells (Figure 4B). Wildtype HPRT1 overexpression also restored the thiopurine conversion in Reh-6MPR cells (Figure 4C, 4D and Supplementary Figure 2), which is consistent with the activity of HPRT1 in those cells (Figure 4E). We also found that expression of wildtype HPRT1 could suppress the de novo purine synthesis pathway up-regulation in the resistant cells (Figure 4F and Supplementary Figure $3 \mathrm{~A})$. On the contrary, re-expression of HPRT1 V165fs mutant shows little effect in thiopurine sensitivity, drug metabolism and purine synthesis regulation (Figure 4B4F and Supplementary Figure 3A). Taking together, our results lead to the assumption that the activity of HPRT1 plays an important role in thiopurine resistance.

\section{Knockdown HPRT1 induce thiopurine resistance}

To further confirm the role of HPRT1 in thiopurine drug resistance, we used CRISPR-Cas9 technology to knockdown endogenous HPRT1 in Reh cells (Figure 5A). The IC50 of 6-MP and 6-TG were dramatically increased in HPRT1 knockdown cells compared to control Reh cells (Figure 5B). Re-expression of wildtype HPRT1 (sgRNA resistent) in the knockdown cells can effectively reversed not only the thiopurine resistant phenotype (Figure 5C and 5D), but also the thiopurine metabolism (Figure 5E, 5F and Supplementary Figure 4). However, reexpression of mutated HPRT1-V165fs (sgRNA resistent) did not show the same effect in thiopurine resistant and thiopurine metabolism (Figure 5C-5F and Supplementary Figure 4). We also used IMP-labeled level to indicate the HPRT1 activity in the re-expression cells and HPRT1V165fs show no enzyme activity (Figure 5G). These results suggest that HPRT1-V165fs is a loss-of-function mutant. Consistent with the observation in resistant cells, de novo purine synthesis pathway was also up regulated when knocking down endogenous HPRT1 (Figure 5H and Supplementary Figure 3B). Expression of wildtype HPRT1 suppressed the increasing of hypoxanthine, but expression of HPRT1-V165fs did not (Figure 5H).

\section{DISCUSSION}

Many mechanisms of modulating thiopurine conversion have been reported: methylation by TPMT, clearance of cytotoxic nucleotides by relapse-specific 
A

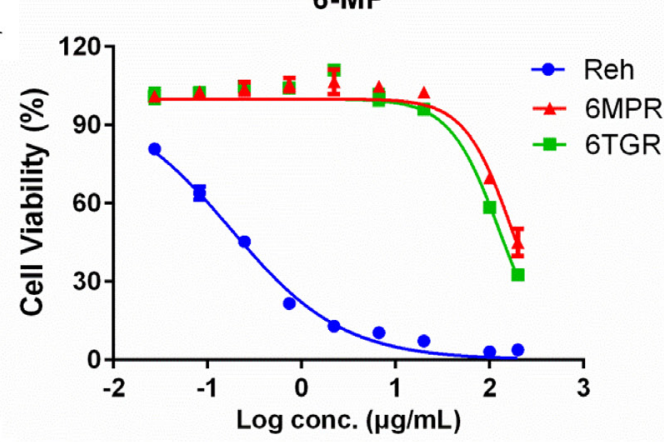

$\mathbf{C}$

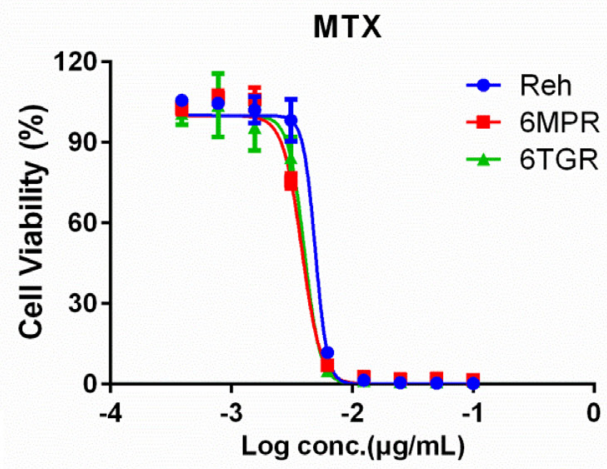

$\mathbf{E}$

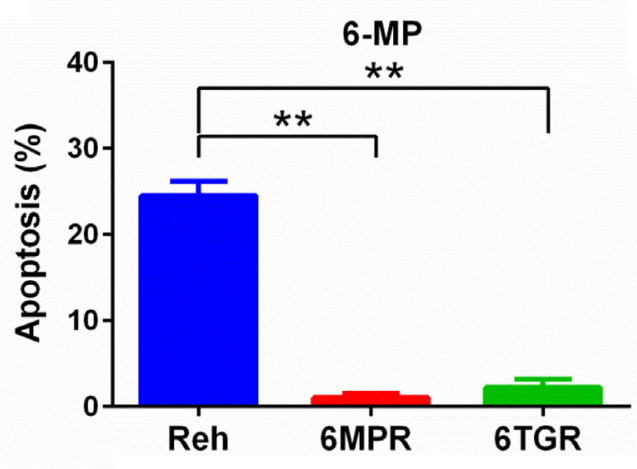

G

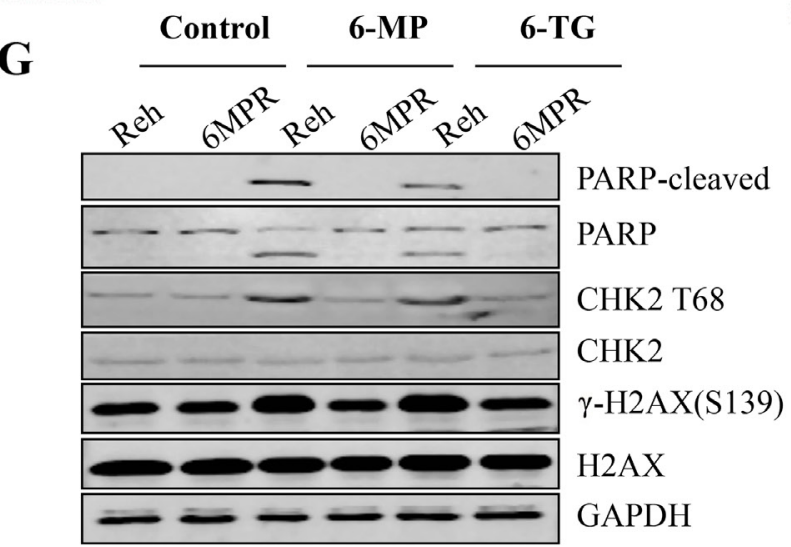

B

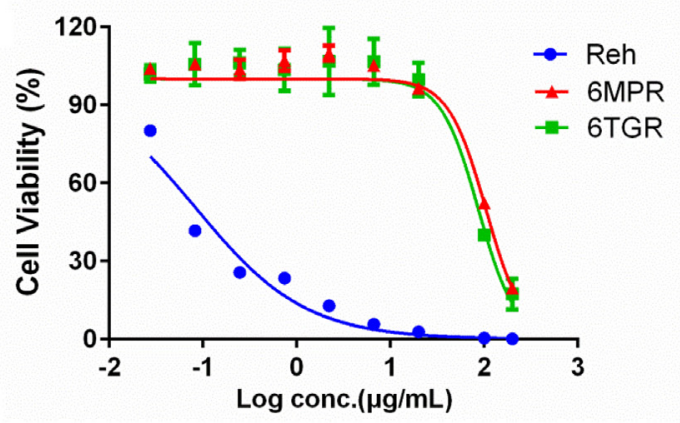

D

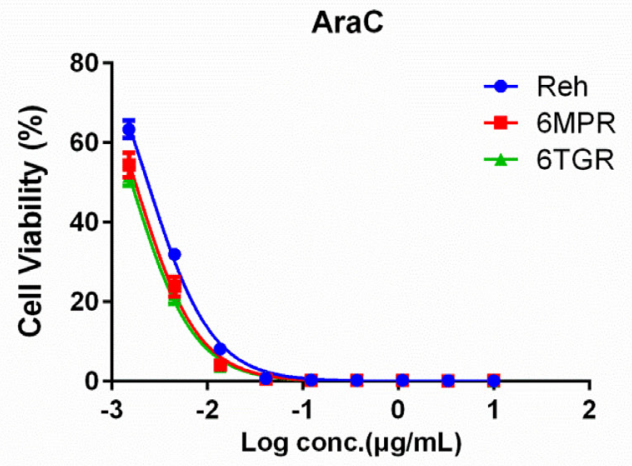

F

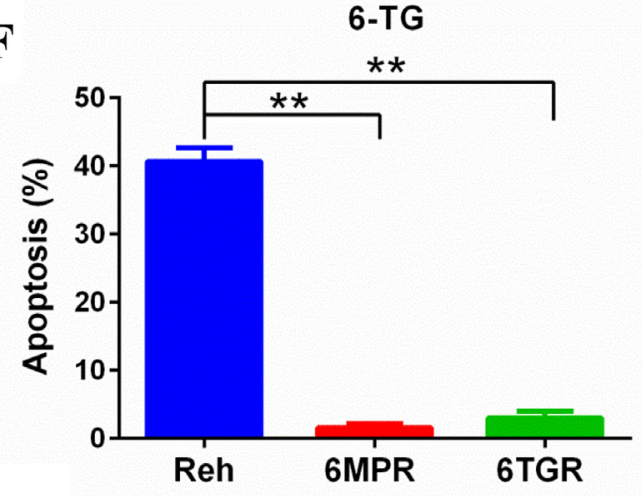

Figure 1: Establishment of the thiopurine resistant cell lines Reh-6MPR and Reh-6TGR. (A, B, C and D) Drug sensitivity of 6-MP, 6-TG, MTX and AraC were analyzed with Cell TiterGlo assay. (E, F) Apoptosis induced by 6-MP and 6-TG was analyzed with annexin V-PE apoptosis detection Kit. Data are expressed as mean $\pm \mathrm{SD} .{ }^{* *} P<0.01$ compared with ctrl Reh group. (G) Western blot of DDR markers (PARP-cleaved, P-CHK2 and $\gamma$-H2AX). 
NT5C2 mutations, competitive inhibition of thiopurine activation by feedback-defective PRPS1 mutations and so on [9-11]. In our study, we did not find TPMT, NT5C2 and PRPS1 mutations in the resistant cell lines, but we identified a novel HPRT1 mutation V165fs. Although many HPRT1 mutations have been found, HPRT1 mutation V165fs was not reported previously. And we identified its loss-of-function mutation and the important role of HPRT1 in thiopurine resistance in ALL through functional analysis.

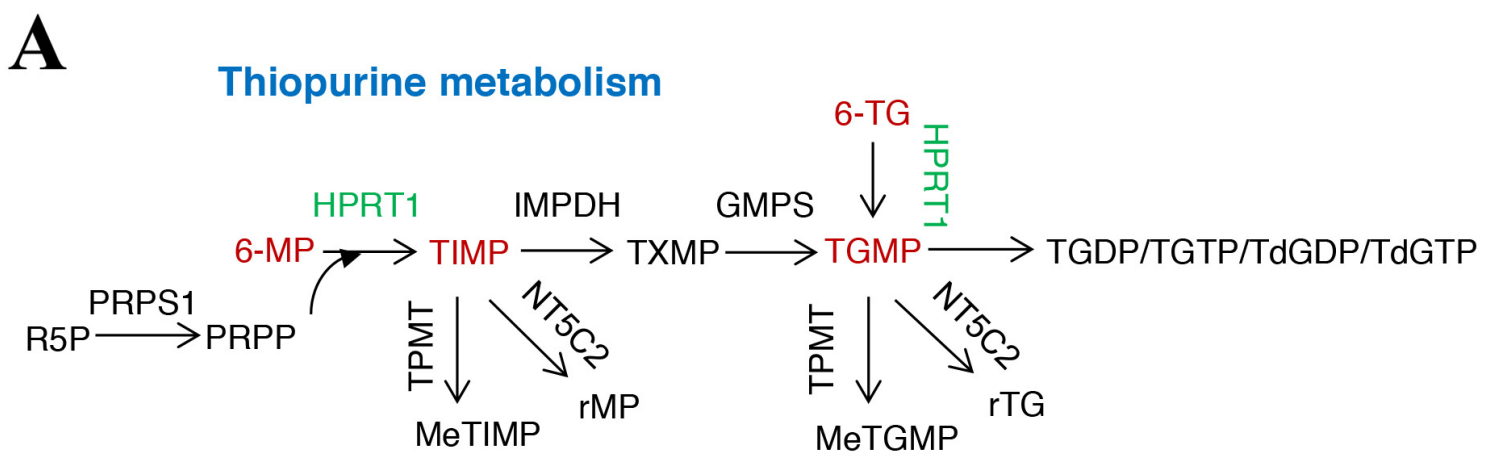

B

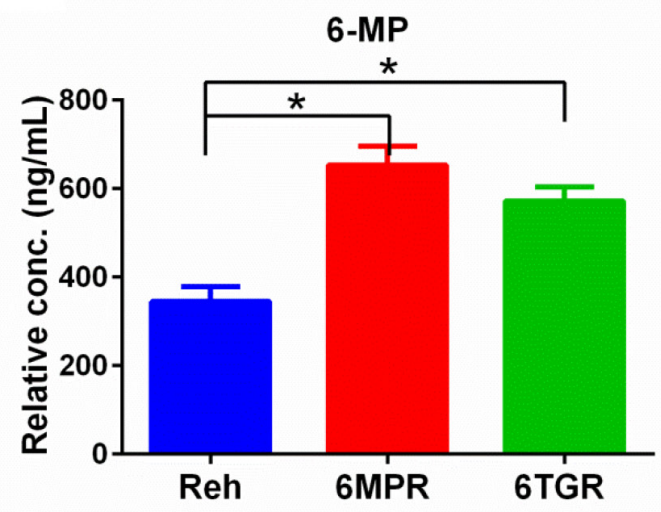

D

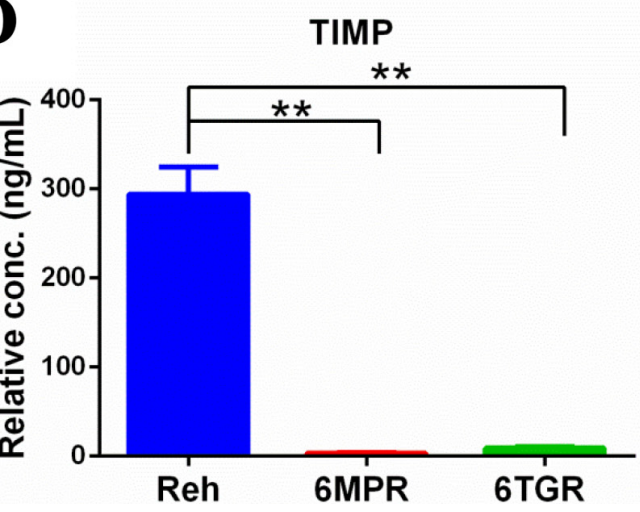

C

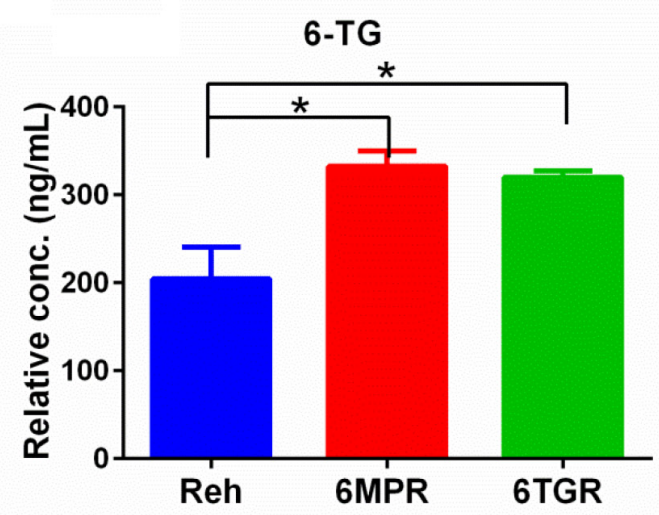

$\mathbf{E}$

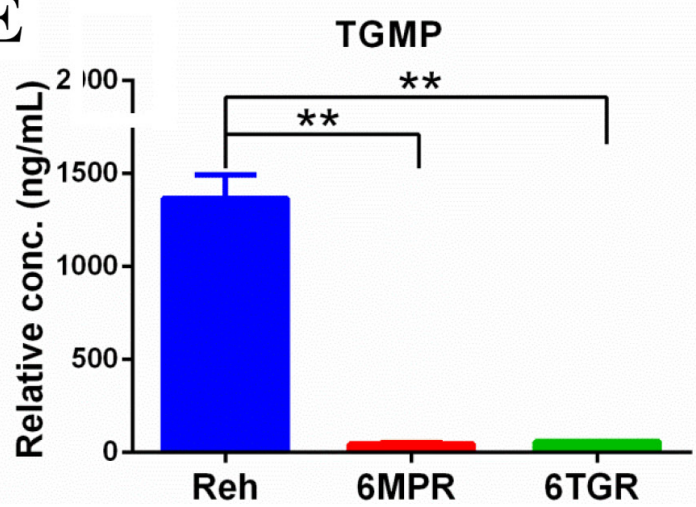

Figure 2: Thiopurine metabolism in Reh cells and resistant cells. (A) The diagram of thiopurine metabolism. (B, C) The accumulations of 6-MP and 6-TG were measured by LC-MS in ctrl Reh cells and resistant cells. Data are expressed as mean \pm SD. ${ }^{*} P<0.05$ compared with ctrl Reh group. (D, E) Drug metabolites (TIMP and TGMP) were measured by LC-MS in ctrl Reh cells and resistant cells. Data are expressed as mean $\pm \mathrm{SD} .{ }^{* *} P<0.01$ compared with ctrl Reh group. 
A
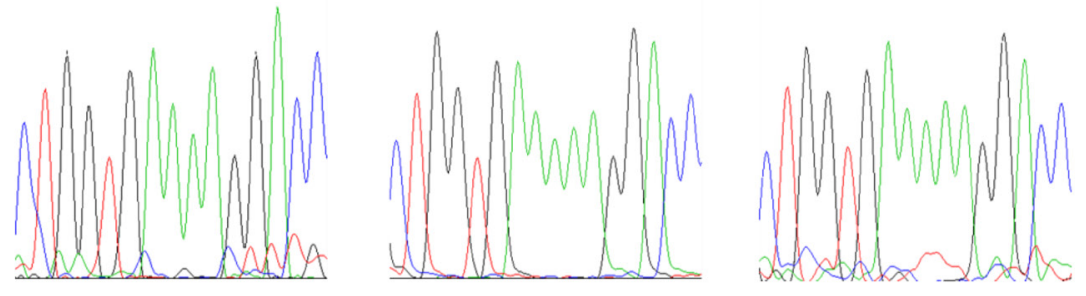

B

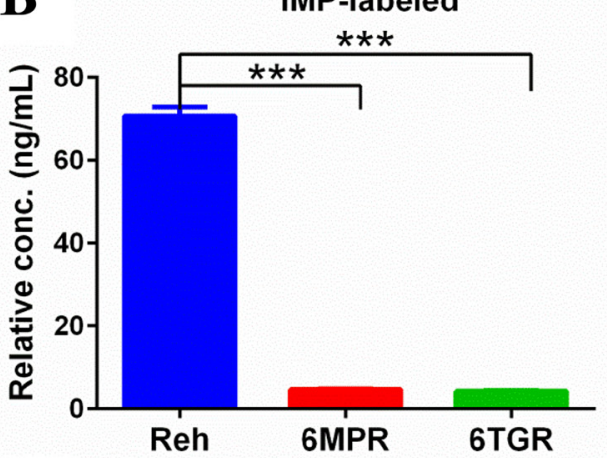

D

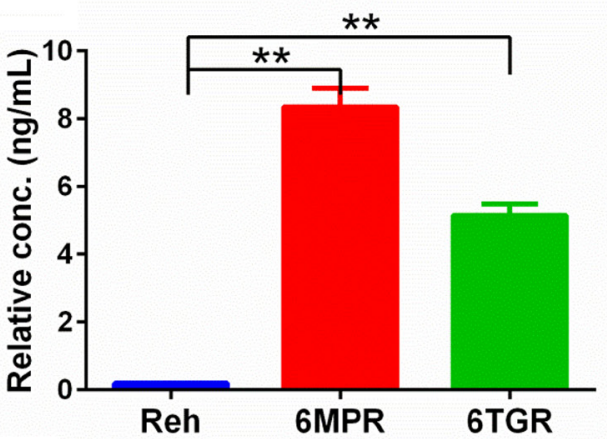

$\mathbf{F}$

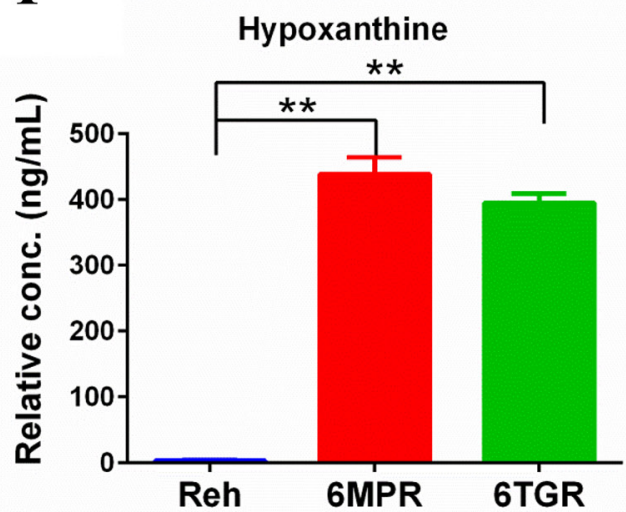

C

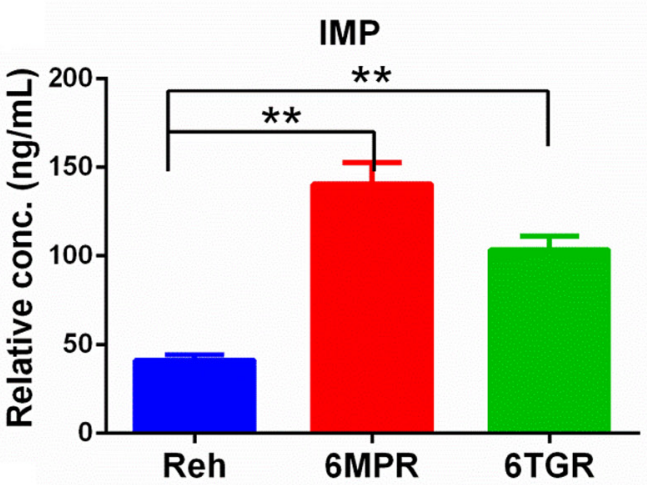

$\mathbf{E}$

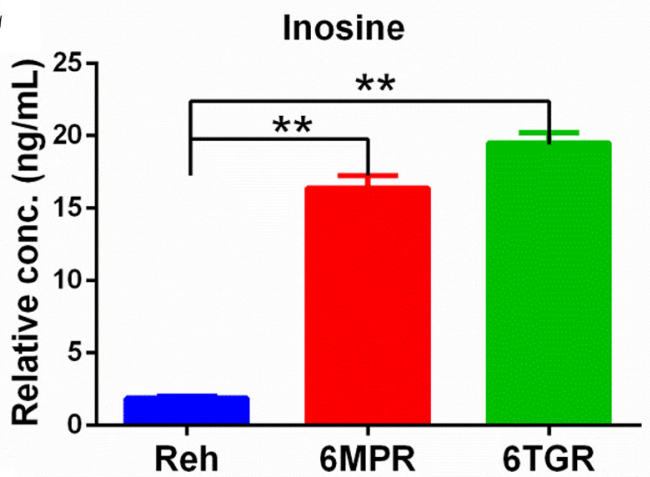

G

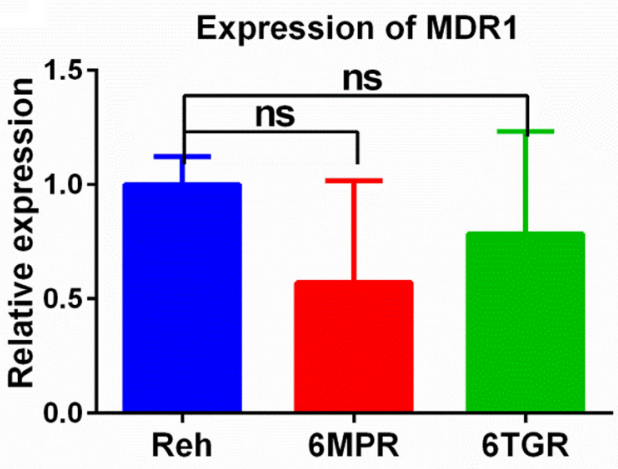

Figure 3: Purine metabolism in Reh cells and resistant cells. (A) Identification HPRT1 mutation in resistant cells by Sanger sequencing. (B) After incubation with $\left[{ }^{13} \mathrm{C}_{5},{ }^{15} \mathrm{~N}_{4}\right]$-hypoxanthine for $4 \mathrm{~h}$, labeled-IMP was measured by LC-MS in Reh cells and resistant cells. Data are expressed as mean \pm SD. ${ }^{* * *} P<0.001$ compared with ctrl Reh group. $(\mathbf{C}, \mathbf{D}, \mathbf{E}, \mathbf{F})$ The metabolites (IMP, AICAR, Inosine and $\mathrm{HX}$ ) were measured by LC-MS in Reh cells and resistant cells. Data are expressed as mean $\pm \mathrm{SD}$. ${ }^{* *} P<0.01$ compared with ctrl Reh group. (G) The expression of MDR1 was detected by using Real-time quantitative RT-PCR assay. Data are expressed as mean $\pm \mathrm{SD}$. 
In thiopurine metabolism, HPRT1 catalyzes the conversion of 6-MP and 6-TG to its active TGNs metabolites [5]. 6-MP and 6-TG were accumulated and its metabolites TIMP and TGMP were markedly decreased in Reh-6MPR cells. Also, the expression

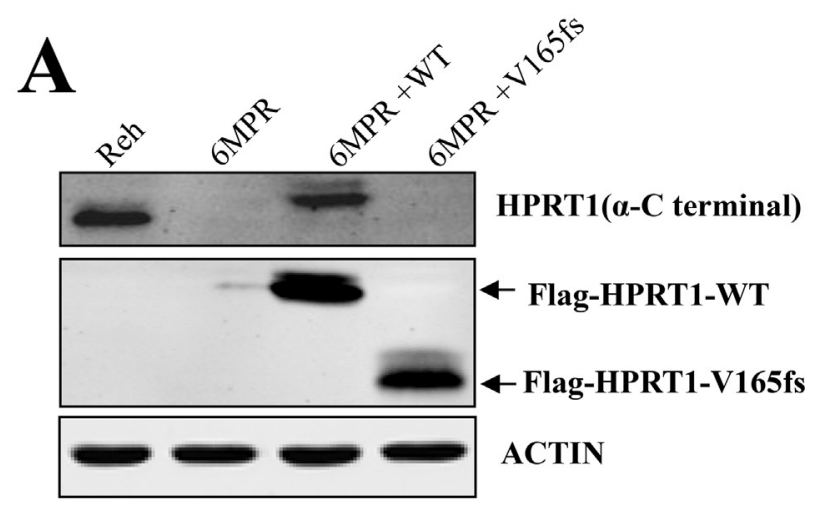

C

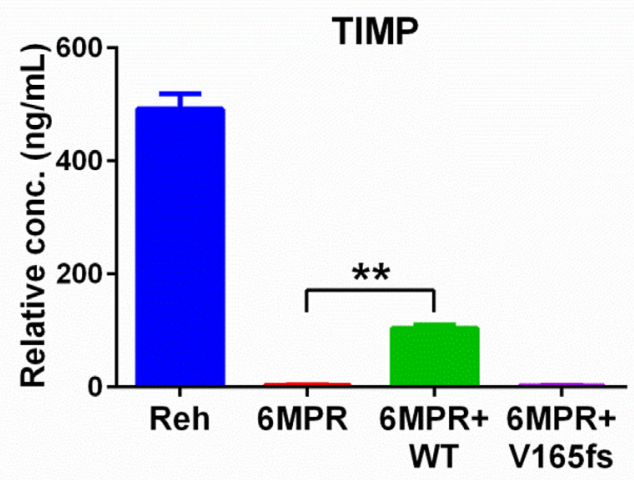

E

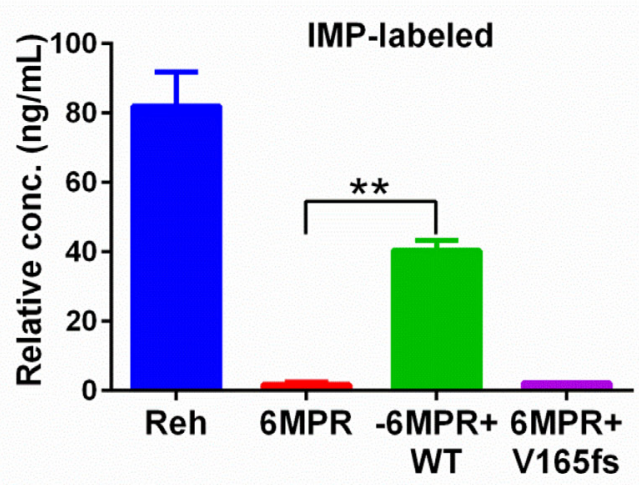

of MDR1 in resistant cells and Reh cells was no significantly different between the two cell lines. These results suggested that the thiopurine uptake is normal but the thiopurine conversion is impaired in the resistant cells.

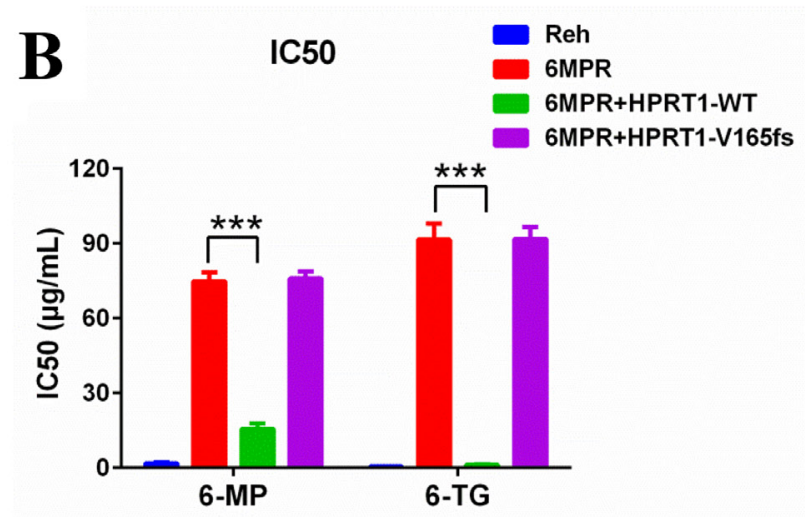

D

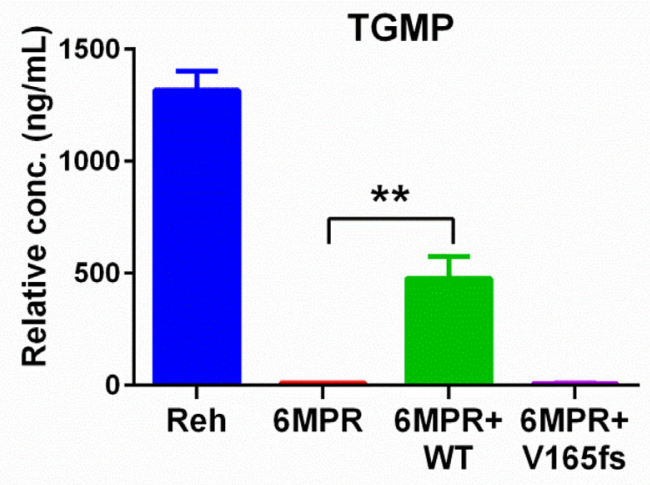

F

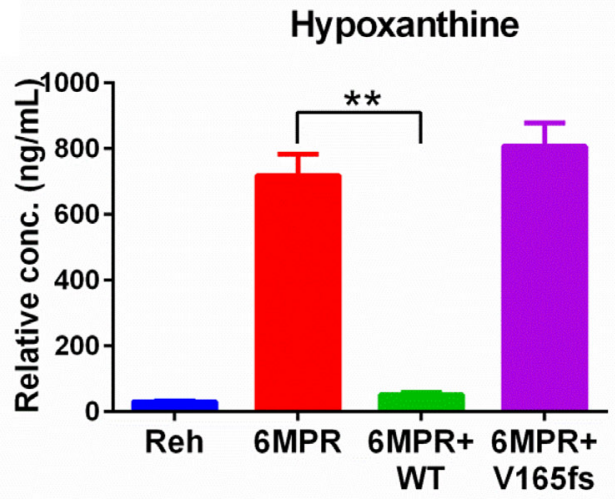

Figure 4: HPRT1-wt can reverse the resistance in Reh-6MPR cells. (A) The expression levels of HRPT1 were detected by western blot. (B) 6-MP and 6-TG IC50 values of Reh-6MPR cells and HPRT1 re-expression cells. Data are expressed as mean \pm SD. ${ }^{* * *} P<0.001$ compared with ctrl Reh group. (C, D, E, F) The TIMP, TGMP, labeled-IMP and hypoxanthine were measured in Reh-6MPR cells and HPRT1 re-expression cells by LC-MS. Data are expressed as mean \pm SD. ${ }^{* *} P<0.01$ compared with ctrl Reh group. 

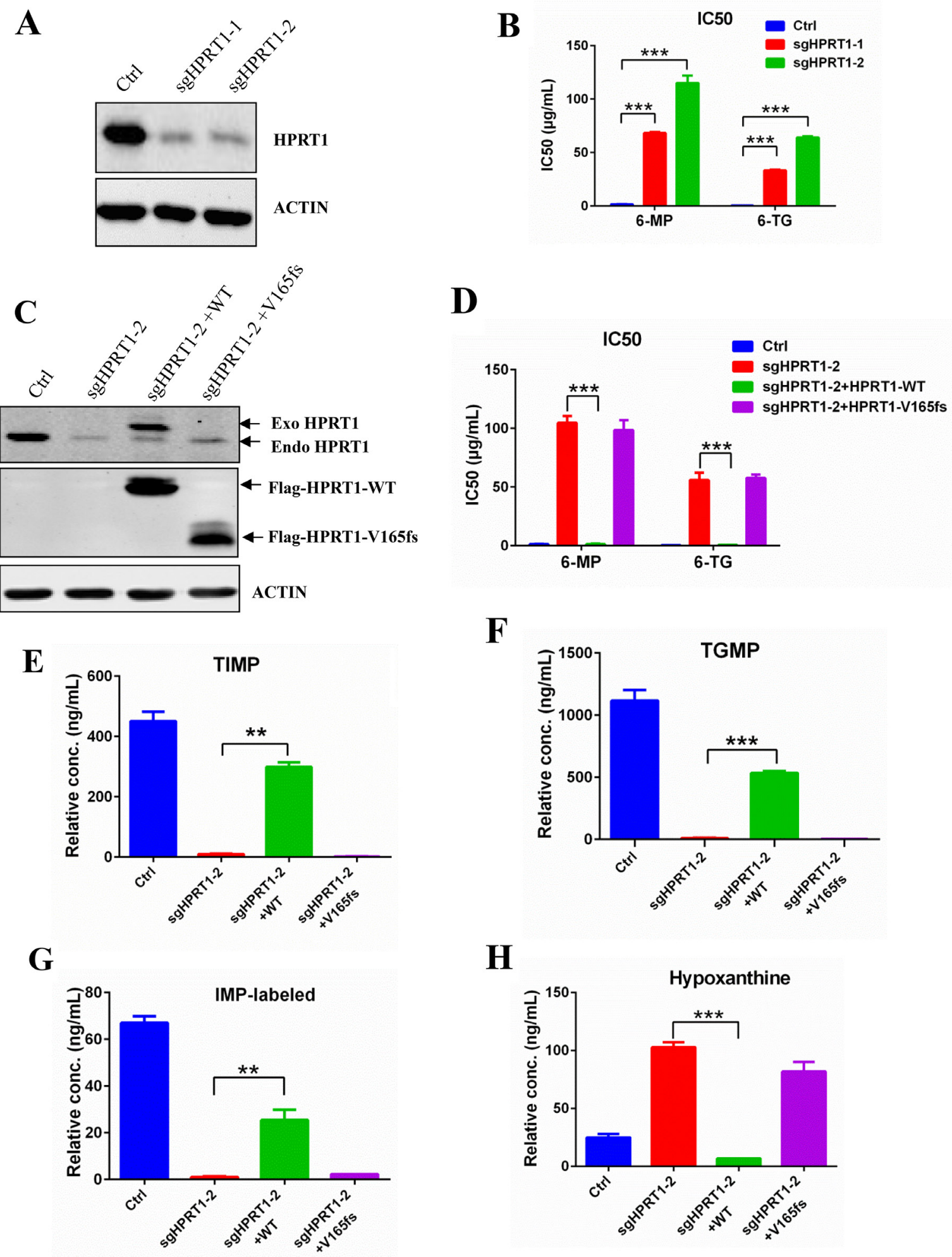

Figure 5: Knockdown HPRT1 induce thiopurine resistance. (A) Western blot of HPRT1 in the knockdown cells. (B) 6-MP and 6-TG IC50 values of HPRT1 knockdown cells. Data are expressed as mean \pm SD. ${ }^{* * *} P<0.001$ compared with ctrl Reh group. (C) The expression levels of endogenous and exogenous HRPT1 were detected by western blot. (D) 6-MP and 6-TG IC50 values of knockdown cells with HPRT1 re-expression. Data are expressed as mean \pm SD. ${ }^{* * *} P<0.001$ compared with ctrl Reh group. (E, F, G, H) The TIMP, TGMP, labeled-IMP and hypoxanthine were measured in HPRT1 knockdown cells and HPRT1 re-expression cells by LC-MS. Data are expressed as mean $\pm \mathrm{SD} .{ }^{* * *} P<0.001,{ }^{* *} P<0.05$ compared with ctrl Reh group. 
HPRT1 is a purine salvage enzyme that catalyzes the conversion of hypoxanthine and phosphoribosyl pyrophosphate (PRPP) to IMP [6]. In thiopurine resistant cells, HPRT1 mutation V165fs failed to convert $\left[{ }^{13} \mathrm{C}_{5},{ }^{15} \mathrm{~N}_{4}\right]$-hypoxanthine to $\left[{ }^{13} \mathrm{C}_{5},{ }^{15} \mathrm{~N}_{4}\right]$-IMP, and when we expressed HPRT1 V165fs mutant in wildtype Reh cells, it could not block the wildtype HPRT1 function (data not shown). These results indicated that the HPRT1-V165fs is a loss-of-function mutation, which excludes the domain negative mechanism.

We observed that the purine biosynthesis intermediates IMP, HX, AICAR and Inosine were increased in the resistant cells, which suggests de novo purine synthesis pathway is up-regulated. The lossof-function mutant HPRT1-V165fs fails to convert thiopurine, meanwhile it impairs the activity of purine salvage pathway in resistant cells. Thus the resistant cells need to up-regulate the de novo purine biosynthesis pathway to supply sufficient nucleotide pools to maintain DNA replication and cell proliferation $[15,16]$.

In conclusion, the present results of this study show that we found the novel HPRT1 mutation V165fs causing loss-of-function of HPRT1 and that the mutation plays a critical role in thiopurine resistance in ALL.

\section{MATERIALS AND METHODS}

\section{Cell culture}

The parental cell line Reh and resistant cell lines Reh-6MPR and Reh-6TGR were cultured in RPMI 1640 medium supplemented with $10 \% \mathrm{FBS}, 100 \mathrm{U} / \mathrm{ml}$ penicillin $\mathrm{G}$ and $100 \mu \mathrm{g} / \mathrm{ml}$ streptomycin. Reh-6MPR and Reh6TGR cells were selected from Reh cells by treating with stepwised increasing concentrations of 6-MP or 6-TG during a 3-month period. All cells were incubated at $37^{\circ} \mathrm{C}$ in $5 \% \mathrm{CO} 2$.

\section{Cell viability}

Drug sensitivity assay was performed as described previously [10]. Briefly, cells were seeded in 96-well plates $(12,000$ cells per well) in $0.1 \mathrm{ml}$ medium and treated for $72 \mathrm{~h}$ with serially diluted anticancer drugs. CellTiterGlo (CellTiter-Glo Luminescent kit, Promega) reagents $(50 \mu \mathrm{l})$ was added to each well and mixed for $10 \mathrm{~min}$ before the luminescence was measured on a microplate reader (Biotek, USA).

\section{Apoptosis assay}

Cells were seeded in triplicate in 24 -well plates $\left(5 \times 10^{5}\right.$ cells per well) and treated for $72 \mathrm{~h}$ with $6-\mathrm{MP}(10 \mu \mathrm{g} / \mathrm{ml})$ or $6-\mathrm{TG}(10 \mu \mathrm{g} / \mathrm{ml})$. Apoptosis was measured by staining with Annexin V-PE and 7-AAD (AnnexinV-PE Apoptosis Detection kit, BD Pharmingen, cat. No.:559763) followed by flow cytometry on a FACS flow cytometer (BD, Canto II).

\section{Analyze of accumulation and metabolites of 6-MP and 6-TG}

Cells were cultured at a density of $5 \times 10^{5}$ cells per $\mathrm{ml}$ for $4 \mathrm{~h}$ in RPMI 1640 containing $10 \mu \mathrm{M} 6-\mathrm{MP}$ or 10 $\mu \mathrm{M}$ 6-TG, then harvested the cells and assayed by a modified method based on what was previously described [10]. Intracellular accumulation of 6-MP and 6-TG and its metabolites (TIMP and TGMP) were determined by LCMS. The relative concentration was defined according to the standard curve of compound dissolved in $80 \%$ methanol.

\section{Purine metabolism in Reh cells and resistant cells}

Purine metabolism assay was performed as described previously [10]. Briefly, cells were cultured in RPMI 1640 at a density of $1 \times 10^{6}$ cells per ml, after addition $\left[{ }^{13} \mathrm{C}_{5},{ }^{15} \mathrm{~N}_{4}\right]$-hypoxanthine (Cambridge Isotope Laboratories, cat. No. CNLM-7894-PK), cells were cultured for an additional $4 \mathrm{~h}$, then harvested and pelleted. The reaction was quenched in cold $80 \%$ methanol, cells were centrifuged at 15,000 r.p.m. for $15 \mathrm{~min}$ and metabolites (IMP, AICAR, Inosine and HX) in the supernatant were analyzed by LC-MS [17]. IMP synthesis through the purine salvage pathway was measured by ${ }^{13} \mathrm{C}_{5},{ }^{15} \mathrm{~N}_{4}$ incorporation (molecular weight peak IMPlabeled); The relative concentrations were defined according to the standard curve of compounds dissolved in $80 \%$ methanol without correcting for cell matrix effect.

\section{Whole-exome sequencing and analysis}

Whole-exome capture libraries were prepared according to standard protocols using SureSelect Human All Exon $50 \mathrm{Mb}$ and $38 \mathrm{Mb}$ kit (Agilent technologies). We performed paired-end $(2 \times 100 \mathrm{bp})$ sequencing using the Illumina HiSeq2000 instruments, imaging analysis and base calling using the Illumina Real Time Analysis (RTA) Pipeline version 1.9. After removing reads with sequence matching the sequencing adaptors and low-quality reads (reads with more than $50 \%$ of bases with Phred quality score of $<5$ ), high-quality reads were aligned to the reference human genome (hg19, http://genome.ucsc.edu/) using Burrows-Wheeler analysis (BWA) with default parameters. The reference human genome assembly hg19 was used for mapping all samples. SNVs were called with GATK and filtered with recommended threshold (SNV quality $\geq 20,>3$ reads covered and depth $\geq 10$ ).

\section{Lentivirus infection and western blot analysis}

The human HPRT1 coding region was cloned into pGV320 mcherry vector (Shanghai GeneChem) and confirmed by DNA sequencing. The lentiviral constructs were packaged as described previously [10]. The virus was transfected into cells supplemented with $8 \mu \mathrm{g} / \mathrm{ml}$ Polybrene 
(Sigma). The medium was changed $24 \mathrm{~h}$ after infection, and cherry-positive cells were sorted with a Moflo XDP (Beckman Counter). Cells were harvested in lysis buffer and analyzed by SDS-PAGE with the following antibodies: antiflag (cat. No.M8823, 1:5,000 dilution, Sigma, USA), $\beta$-actin (cat. No. M1210-2, 1:5,000 dilution, HuaAn Biotechnology, China), HPRT1 (cat. No.109021, 1:10,000 dilution, Abcam, UK). The Reh and resistant cells were treated with 6-MP (1 $\mu \mathrm{g} / \mathrm{ml})$ or $6-\mathrm{TG}(1 \mu \mathrm{g} / \mathrm{ml})$ for $48 \mathrm{~h}$ and analyzed by SDSPAGE with the following antibodies: $\gamma$-H2AX (S139) (cat. 9718; 1:1,000 dilution, Cell Signaling Technology (CST), USA), H2AX (cat. 7631; 1:1,000 dilution, CST, USA), phospho-CHK2 (T68) (cat. 1538-1; 1:1,000 dilution, CST, USA), CHK2 (cat. 3428-1; 1:50,000 dilution, Epitomics, USA), PARP (cat. 9542; 1:1,000 dilution, CST, USA), cleaved PARP (cat. 5625; 1:1,000 dilution, CST, USA) and GAPDH (cat. No. R1208-3, 1:5,000 dilution, HuaAn Biotechnology, China). Immunoblots were analyzed using the Odyssey system (LI-COR Biosciences, USA).

\section{Real-time quantitative RT-PCR assay}

Total cellular RNA was isolated from cells using trizol extraction method. For RT-PCR, $1 \mu \mathrm{g}$ total RNA of the sample was used for cDNA synthesis by using PrimeScript ${ }^{\mathrm{TM}} \mathrm{RT}$ reagent kit with gDNA Eraser (Takara, cat. No. RR047A). The primer sequence of MDR1 was: sense 5'-CGACAGGAGATAGGCTGGTT-3' and antisense 5'- GGTTAGCTTCCAACCACGTG-3'. The primer sequence of the internal control actin was: sense 5'-TGACGTGGACATCCGCAAAG-3' and antisense 5'-CTGGAAGGTGGACAGCGAGG-3'. Real-time quantitative PCR was carried out for 40 cycles as follows: initial denaturation at $94^{\circ} \mathrm{C}$ for $5 \mathrm{~min}$, template denaturation at $94^{\circ} \mathrm{C}$ for $30 \mathrm{sec}$, primer annealing at $60^{\circ} \mathrm{C}$ for $30 \mathrm{sec}$ and primer extension/elongation at $72^{\circ} \mathrm{C}$ for 30 sec. The relative expression (RE) was calculated by the $2^{-\Delta \Delta \mathrm{Ct}}$ method [18].

\section{Statistical analysis}

Each result was repeated at least three times. The data were expressed as mean $\pm \mathrm{SD}$ and analyzed by the Student's $t$-test, $\mathrm{P}<0.05$ was considered to be statistically significant.

\section{Author contributions}

H.L., B.-B.S.Z. and Y.Z. designed the research; F.Y., H.S.F., Y.C., and D.W. performed experiments; F.Y., H.S.F., H.L. analyzed the data and wrote the manuscript.

\section{ACKNOWLEDGMENTS AND FUNDING}

We thank other members of the lab for their assistances. This work was supported by grants from the National Natural Science Foundation of China (81500112, 81372349, and 31530017) and the Science and Technology Commission of Pudong New Area Foundation (PKJ2016-Y37).

\section{CONFLICTS OF INTEREST}

The authors declare no conflicts of interest.

\section{REFERENCES}

1. Pui CH, Evans WE. Treatment of acute lymphoblastic leukemia. N Engl J Med. 2006; 354:166-78. https://doi. org/10.1056/NEJMra052603.

2. Einsiedel HG, von Stackelberg A, Hartmann R, Fengler R, Schrappe M, Janka-Schaub G, Mann G, Hahlen K, Gobel U, Klingebiel T, Ludwig WD, Henze G. Long-term outcome in children with relapsed ALL by risk-stratified salvage therapy: results of trial acute lymphoblastic leukemiarelapse study of the Berlin-Frankfurt-Munster Group 87. J Clin Oncol. 2005; 23:7942-50. https://doi.org/10.1200/ JCO.2005.01.1031.

3. Pui CH, Carroll WL, Meshinchi S, Arceci RJ. Biology, risk stratification, and therapy of pediatric acute leukemias: an update. J Clin Oncol. 2011; 29:551-65. https://doi. org/10.1200/jco.2010.30.7405.

4. Rivera GK, Zhou Y, Hancock ML, Gajjar A, Rubnitz J, Ribeiro RC, Sandlund JT, Hudson M, Relling M, Evans WE, Pui CH. Bone marrow recurrence after initial intensive treatment for childhood acute lymphoblastic leukemia. Cancer. 2005; 103:368-76. https://doi.org/10.1002/ cncr.20743.

5. Karran P, Attard N. Thiopurines in current medical practice: molecular mechanisms and contributions to therapyrelated cancer. Nat Rev Cancer. 2008; 8:24-36. https://doi. org/10.1038/nrc2292.

6. Ding L, Zhang FB, Liu H, Gao X, Bi HC, Wang XD, Chen BL, Zhang Y, Zhao LZ, Zhong GP, Hu PJ, Chen MH, Huang M. Hypoxanthine guanine phosphoribosyltransferase activity is related to 6-thioguanine nucleotide concentrations and thiopurine-induced leukopenia in the treatment of inflammatory bowel disease. Inflamm Bowel Dis. 2012; 18:63-73. https://doi.org/10.1002/ibd.21676.

7. Jinnah HA, De Gregorio L, Harris JC, Nyhan WL, O’Neill JP. The spectrum of inherited mutations causing HPRT deficiency: 75 new cases and a review of 196 previously reported cases. Mutat Res. 2000; 463:309-26.

8. Ogasawara N. [Hypoxanthine guanine phosphoribosyl transferase]. [Article in Japanese]. Nihon Rinsho. 1996; 54:3207-12.

9. Hartford C, Vasquez E, Schwab M, Edick MJ, Rehg JE, Grosveld G, Pui CH, Evans WE, Relling MV. Differential effects of targeted disruption of thiopurine methyltransferase on mercaptopurine and thioguanine 
pharmacodynamics. Cancer Res. 2007; 67:4965-72. https:// doi.org/10.1158/0008-5472.CAN-06-3508.

10. Li B, Li H, Bai Y, Kirschner-Schwabe R, Yang JJ, Chen Y, Lu G, Tzoneva G, Ma X, Wu T, Li W, Lu H, Ding L, et al. Negative feedback-defective PRPS1 mutants drive thiopurine resistance in relapsed childhood ALL. Nat Med. 2015; 21:563-71. https://doi.org/10.1038/nm.3840.

11. Tzoneva G, Perez-Garcia A, Carpenter Z, Khiabanian H, Tosello V, Allegretta M, Paietta E, Racevskis J, Rowe JM, Tallman MS, Paganin M, Basso G, Hof J, et al. Activating mutations in the NT5C2 nucleotidase gene drive chemotherapy resistance in relapsed ALL. Nat Med. 2013; 19:368-71. https://doi.org/10.1038/nm.3078.

12. Keough DT, Brereton IM, de Jersey J, Guddat LW. The crystal structure of free human hypoxanthine-guanine phosphoribosyltransferase reveals extensive conformational plasticity throughout the catalytic cycle. J Mol Biol. 2005; 351:170-81. https://doi.org/10.1016/j.jmb.2005.05.061.

13. Peng XX, Shi Z, Tiwari AK, Damaraju VL, Fu L, Cass CE, Ashby CR Jr, Kruh GD, Chen ZS. Up-regulation of P-glycoprotein confers acquired resistance to 6-mercaptopurine in human chronic myeloid leukemia cells. Oncol Lett. 2011; 2:549-56. https://doi.org/10.3892/ol.2011.288.
14. Peng XX, Tiwari AK, Wu HC, Chen ZS. Overexpression of P-glycoprotein induces acquired resistance to imatinib in chronic myelogenous leukemia cells. Chin J Cancer. 2012; 31:110-8. https://doi.org/10.5732/cjc.011.10327.

15. Tong $\mathrm{X}$, Zhao F, Thompson CB. The molecular determinants of de novo nucleotide biosynthesis in cancer cells. Curr Opin Genet Dev. 2009; 19:32-7. https://doi. org/10.1016/j.gde.2009.01.002.

16. Bester AC, Roniger M, Oren YS, Im MM, Sarni D, Chaoat M, Bensimon A, Zamir G, Shewach DS, Kerem B. Nucleotide deficiency promotes genomic instability in early stages of cancer development. Cell. 2011; 145:435-46. https://doi.org/10.1016/j.cell.2011.03.044.

17. Dervieux T, Chu Y, Su Y, Pui CH, Evans WE, Relling MV. HPLC determination of thiopurine nucleosides and nucleotides in vivo in lymphoblasts following mercaptopurine therapy. Clin Chem. 2002; 48:61-8.

18. Livak KJ, Schmittgen TD. Analysis of relative gene expression data using real-time quantitative PCR and the 2(-Delta Delta C(T)) method. Methods. 2001; 25:402-8. https://doi.org/10.1006/meth.2001.1262. 